\title{
On the Path Coverage Properties of Random Sensor Networks
}

\author{
S. Sundhar Ram, Student Member, IEEE, D. Manjunath, Member, IEEE, \\ Srikanth K. lyer, and D. Yogeshwaran
}

\begin{abstract}
In a sensor network, the points in the operational area that are suitably sensed are a two-dimensional spatial coverage process. For randomly deployed sensor networks, typically, the network coverage of two-dimensional areas is analyzed. However, in many sensor network applications, e.g., tracking of moving objects, the sensing process on paths, rather than in areas, is of interest. With such an application in mind, we analyze the coverage process induced on a one-dimensional path by a sensor network that is modeled as a two-dimensional Boolean model. In the analysis, the sensor locations form a spatial Poisson process of density $\lambda$ and the sensing regions are circles of i.i.d. random radii. We first obtain a strong law for the fraction of a path that is $k$-sensed, i.e., sensed by $(\geq k)$ sensors. Asymptotic path-sensing results are obtained under the same limiting regimes as those required for asymptotic coverage by a two-dimensional Boolean model. Interestingly, the asymptotic fraction of the area that is 1 -sensed is the same as the fraction of a path that is 1 -sensed. For $k=1$, we also obtain a central limit theorem that shows that the asymptotics converge at the rate of $\Theta\left(\lambda^{1 / 2}\right)$ for $k=1$. For finite networks, the expectation and variance of the fraction of the path that is $k$-sensed is obtained. The asymptotics and the finite network results are then used to obtain the critical sensor density to $k$-sense a fraction $\alpha_{k}$ of an arbitrary path with very high probability is also obtained. Through simulations, we then analyze the robustness of the model when the sensor deployment is nonhomogeneous and when the paths are not rectilinear. Other path coverage measures like breach, support, "length to first sense," and sensing continuity measures like holes and clumps are also characterized. Finally, we discuss some generalizations of the results like characterization of the coverage process of $m$-dimensional "straight line paths" by $n$-dimensional, $n>m$, sensor networks.
\end{abstract}

Index Terms-Sensor networks coverage, path tracking, Boolean models, exposure.

\section{INTRODUCTION}

SENSOR networks are formed from a large number of $S$ randomly deployed sensor nodes. These sensor nodes sense a phenomenon, possibly process the collected sensing data in a collaborative manner, and route the results to an end user. The phenomenon that is being sensed could be a localized event, e.g., an acoustic point source, or it could be a spatial phenomenon spread throughout the operational area of the sensor network, e.g., target tracking and atmospheric monitoring. Each sensor node will have a footprint over which it can perform the measurements and a random sensor network may not sense the entire operational area. The accuracy of processing the sensor network depends on its sensing ability.

Consider a sensor network for target tracking. A typical trajectory estimation algorithm for tracking of a moving target would work as follows: Whenever the target can be sensed by a sufficient number of sensors, point estimates of

- S.S. Ram is with the Department of Electrical and Computer Engineering, University of Illinois at Urbana-Champaign, Champaign, IL. E-mail ssriniv5@uiuc.edu.

- D. Manjunath is with the Department of Electrical Engineering, Indian Institute of Technology, Bombay, India. E-mail dmanju@ee.iitb.ac.in.

- S.K. Iyer and D. Yogeshwaran are with the Department of Mathematics, Indian Institute of Science, Bangalore, India.

E-mail: \{skiyer, yogesh\}@math.iisc.ernet.in.

Manuscript received 1 Jan. 2006; revised 30 Apr. 2006; accepted 14 Sept. 2006; published online 7 Feb. 2007.

For information on obtaining reprints of this article, please send e-mail to: tmc@computer.org, and reference IEEECS Log Number TMC-0002-0106.

Digital Object Identifier no. 10.1109/TMC.2007.1000. the location are obtained. These estimates are then appropriately filtered to estimate the trajectory for the times when the target is not sufficiently sensed. The quality of the trajectory estimates will depend on the fraction of the trajectory that is being sensed by a specified minimum number of sensors, which therefore is a measure of the tracking ability, or trackability, of the sensor network. If the complete trajectory is not being sufficiently sensed, then an immediate measure of trackability is the "length to first sense," i.e., the distance traveled by the target in the operational area before it is sensed. This can also be interpreted as the time to detect an intruder in an intrusion detection network. Another measure of trackability would be the length of a continuous segment that is tracked by a given number (or a given minimum number) of sensors, a measure of the "sensing continuity." We can see that the above properties are indicators of the accuracy with which the network can track the target, i.e., the trackability of the network.

Notice that the trackability measures that we discussed above are essentially statistics of the coverage of a path rather than the coverage of an area. Thus, in measuring the trackability of a random sensor network, we need to obtain the coverage induced on a one-dimensional path by a twodimensional coverage process. This is the focus of this paper-we analyze the path-coverage induced by an area coverage process in a random sensor network and obtain the trackability measures defined here. In addition to obtaining the aforementioned measures, after formally 
defining them, we also obtain other measures that have been defined in the literature, like breach and support [1].

It is to be noted that this paper is not about tracking an object as it moves in the operational area of the sensor network. The literature on tracking, e.g., [2], [3], has an algorithmic flavor and the objective in such papers is to estimate the position of a target (source) as a function of time. In contrast, our interest in this paper is in trackability, i.e., in knowing if a sensor network will sense a path, possibly for tracking an object moving on it (or an event that is represented by points in a one-dimensional set), as opposed to measure some attribute of the event-we just want to know if we can measure it at all! It may be noted here that characterizing a tracking algorithm requires analysis that will be based on the accuracy of the measurement. Thus, this paper is in the same spirit as those that study area coverage and path sensing, e.g., [4], [5], [6], [7] and not along the lines of those that provide tracking algorithms or analysis, e.g., [2].

We use the following sensing model: The sensors are deployed according to a spatial Poisson process. The sensing area of each sensor is a circle of random radius and a point is considered sensed if and only if it is in the sensing area of at least $k$ sensors. Thus, the coverage of the operational area by the sensors is a two-dimensional coverage process of a Boolean model. (For brevity, we will call this coverage process the two-dimensional Boolean process.) We analyze the properties of the coverage process on an arbitrary straight line path. Thus, our interest is in the statistical properties of the coverage of a one-dimensional path induced by a two-dimensional coverage process of the sensors. The trackability measures are essentially the coverage statistics of this one-dimensional process. While the straight line path was chosen for simplicity, the asymptotic scaling laws that we derive are applicable to curvilinear paths too.

The area coverage properties have been extensively studied in the literature, most notably in [8]. The properties of the induced one-dimensional process seem to have not received the same attention and we develop a method to analyze such a process. To the best of our knowledge, this is the first such analysis. The two are clearly intimately related because nontrivial coverage of the two-dimensional region will be required to obtain nontrivial coverage of onedimensional paths. However, the nature of the relationship is not clear and we explore it in this paper. We will obtain asymptotic results for the one-dimensional path process under the same limiting regime as those required for obtaining nontrivial coverage results for a two-dimensional area process.

\subsection{Summary of Results and Organization of the Paper}

We formally define the trackability measures and discuss related literature in Section 2. We then prove in Section 3 that the sensing process on any straight line path $L$ in the two-dimensional Boolean field is a one-dimensional Boolean process or, equivalently, a $M / G / \infty$ queue.

Using this, we obtain asymptotic and finite network results for the fraction of a straight line path that is $k$-sensed i.e., sensed by $k$ or more sensors, in Section 4 . We first obtain a strong law for the fraction of a path that is $k$-sensed. The asymptotic results are obtained under the same limiting regimes as those required for asymptotic coverage by a twodimensional Boolean process. Interestingly, the asymptotic fraction of the path that is $k$-sensed is the same as the fraction of the area that is $k$-covered by the sensors. Clearly, this equality needs to be proved. For the case when $k=1$, we complement the strong law with a central limit theorem. Based on this, we conclude that, for $k=1$, the asymptotics converge at the rate of $\Theta\left(\lambda^{1 / 2}\right)$, where $\lambda$ is the sensor density. This convergence rate is different from that of area coverage. For finite networks, expectation and variance of the fraction of the path that is $k$-sensed is obtained where, once again, we see that the second order statistics are different from that of area coverage. We then obtain a critical sensor density to $k$-sense a fraction $\alpha_{k}$ of an arbitrary path with very high probability. Through simulations, we also extend this result to weakly nonhomogeneous fields and curvilinear paths.

Other trackability measures like "length to first sense" and sensing continuity measures like holes and clumps are characterized in Section 5. Measures that do not depend on the sensing radius like breach and support are characterized in Section 6. These measures cannot be obtained from a area coverage analysis. Finally, we discuss some generalizations of the results like characterization of the coverage process of $m$-dimensional "straight line paths" by an $n(>m)$-dimensional sensor network in Section 7. We conclude with a brief discussion in Section 8 .

\section{Performance Measures and System Model}

Let $\Omega$ be the operational area of the sensor network and let $\left\{s_{i}\right\}$ be the set of sensors with sensor $s_{i}$ located at $X_{i} \in \Omega$. The following two measures of the "goodness" of deployment with respect to sensing a path are defined in [1]. For a given deployment and a path $L \subset \Omega$, the breach of $L, \operatorname{Br}(L)$, is defined as

$$
\min _{i} \min _{x \in L}\left\|X_{i}-x\right\|
$$

and the support for the path $L, S u(L)$, is defined as

$$
S u(L)=\max _{x \in L}\left\|x-X_{i *}\right\| .
$$

Here, $X_{i *}$ is the location of the sensor closest to path $L$ and the norms above are the euclidean norms. Observe that these measures are independent of the sensing radius. We will obtain the mean and variance of breach and support in Section 6 .

To develop other trackability measures, we first define the sensing process. For every $\left(s_{i}, x\right), x \in \Omega$, the sensing function, $\phi\left(s_{i}, x\right)$, captures the ability of sensor $s_{i}$ to sense a target at point $x$. Note that $\phi\left(s_{i}, x\right)$ could be a random variable. This leads us to define a sensor intensity function

$$
\psi(x, \theta, \phi)= \begin{cases}1 & \text { if } \mathcal{V}\left(\phi\left(s_{1}, x\right), \phi\left(s_{2}, x\right), \ldots\right) \geq \theta \\ 0 & \text { otherwise }\end{cases}
$$


Here, $\mathcal{V}$ is some operator and $\theta$ is some constant. $\psi(x, \theta, \phi)$ captures the summary effect of all the sensors at point $x$ and we consider a point to be sensed only when $\psi(x, \theta, \phi)=1$.

$\phi(\cdot)$ and $\psi(\cdot)$ defined above lead us to the next measure of trackability that we consider in this paper-exposure of a path $L$.

Definition 1. The exposure, $\mathrm{X}_{\psi}(L)$, of a path $L$ in $\Omega$ is the

$$
\mathrm{X}_{\psi}(L)=\frac{\int_{x \in L} \psi(x, \theta, \phi) d x}{|L|},
$$

where $|L|$ denotes the length of the path $L$. This is essentially the same as that defined in [9] except that we also normalize it to the length of the path.

In this paper, we will primarily discuss thresholded sensing, where we assume that sensor $s_{i}$ has a random sensing radius $R_{i}$ within which it can sense perfectly and beyond which it cannot sense, i.e., if the location of the $s_{i}$ is $X_{i}$, then

$$
\phi_{T}\left(s_{i}, x\right)= \begin{cases}1 & \text { if }\left\|x-X_{i}\right\| \leq R_{i} \\ 0 & \text { otherwise. }\end{cases}
$$

The subscript $T$ refers to thresholded sensing. Further, we can specialize thresholded sensing into $k$-thresholded sensing, where we define

$$
\psi_{T}(x, k)= \begin{cases}1 & \text { if } \sum_{i} \phi_{T}\left(s_{i}, x\right) \geq k \\ 0 & \text { otherwise. }\end{cases}
$$

Here, point $x$ is sensed only if it is in the sensing range of at least $k$ sensors. An example of the use of such a sensor intensity function is in position localization that requires range estimates from at least three sensors.

Observe that when the sensor locations form a random process and the sensing radii are random, $\left\{\sum_{i} \phi_{T}\left(s_{i}, x\right)>\right.$ $k\}$ will be a random event and, hence, $\left\{\psi_{T}(x ; k)\right\}_{x \in L}$ will be a random process. The measures of trackability which we define next are essentially statistics of this random process. Thus, exposure is the fraction of the path that will be sensed. A measure of sensing continuity is a clump that is defined as follows for this model:

Definition 2. For $k$-thresholded sensing, a clump on a path $L$ is a contiguous segment of $L$ for which $\psi_{T}(x, k)=1$. Any segment of $L$ between two consecutive clumps is a hole.

The length to first sense can be defined as follows:

Definition 3. For $k$-thresholded sensing, we define the lengthto-first-sense for a path $L, L F(L)$, as the distance to the first point on $L$ where $\psi_{T}(x, k)=1$. We will say that $L F(L)=l_{0}$ if $L$ is not sensed, $l_{0}$ being the length of $L$.

The above notions are illustrated in Fig. 1.

When $\phi\left(s_{i}, x\right)$ is a continuous function, the sensing process is said to be nonthresholded. The trackability measures for the nonthresholded sensing can be lower bounded by the measures for an appropriately defined thresholded sensing case. We discuss this connection in detail in Section 8.

We let $\Omega$ be $\Re^{2}$ and $\left\{X_{i}\right\}$, the set of sensor locations, form a spatial Poisson process in $\Re^{2}$ of density $\lambda$. An excellent

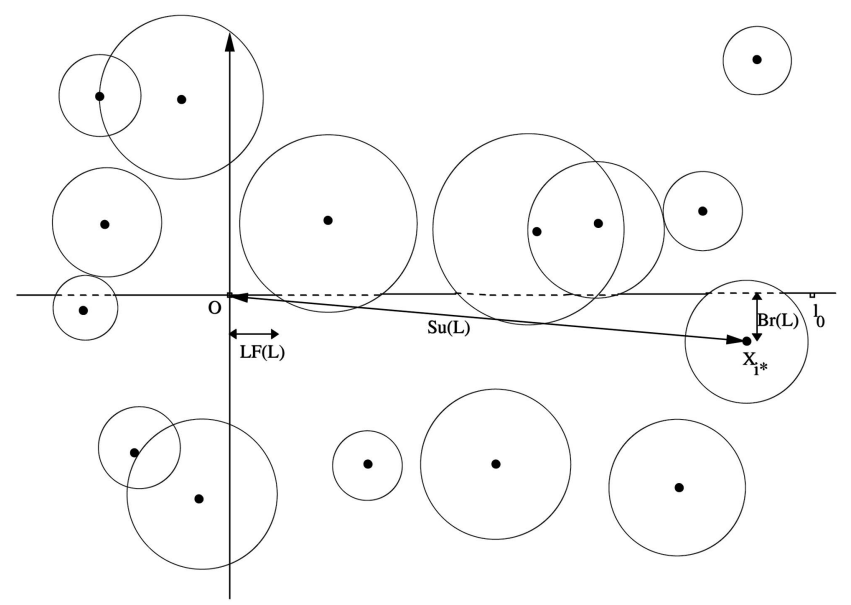

Fig. 1. The figure shows an instance of a sensor network that performs thresholded sensing. The dots represent the sensors and the circle is its sensing area. Path $L$ is the segment $\left[0, l_{0}\right]$. The dotted parts of $L$ are the clumps on it and the thick parts are its holes. $L F(L)$ is the length to its first 1 -sense, $X_{i *}$ the location of the closest sensor to $L, \operatorname{Br}(L)$ is its breach, and $S u(L)$ is its support.

discussion on the physical interpretations of this model is available in [8]. Further, the Poisson process has been extensively used to model the sensor locations, e.g., [4], [5]. $\left\{R_{i}\right\}_{\{i>0\}}$ is assumed to be a sequence of positive i.i.d. random variables whose density has bounded (or compact) support. Without loss of generality, we assume the support to be $[0,1]$. Let $f_{R_{i}}(r)$ denote the density of $R_{i}$ and let $\beta:=\mathrm{E}\left(R_{i}\right)$. Therefore, $\psi_{T}(x, k), x \in \Re^{2}$, is the coverage process formed by placing random circles in $\Re^{2}$ with the location of the circle centers drawn from a spatial Poisson process. This is a special case of the two dimensional Boolean process or the "germ-grain" process studied in [5], [8], [10]. Formally,

Definition 4. An n-dimensional Boolean process is a collection of random sets $X_{i}+C_{i}$, where $X_{i} \in \Re^{n}$ are the points of a Poisson point process, $C_{i}$ are identically distributed, independent random subsets of $\Re^{n}$, and $X_{i}+C_{i} \equiv\left\{X_{i}+x: x \in C_{i}\right\}$ [8].

However, as we have mentioned earlier, our interest in this paper is to study the properties of $\psi_{T}(x, k), x \in L$, where $L$ is an arbitrary straight line path in $\Omega$. These properties depend on the statistics of one-dimensional sets embedded in a two-dimensional space. We mention here that, although much of the paper is on sensor networks in $\Re^{2}$, extensions of some of the results to bounded $\Omega$ are discussed in Section 7.

Prior work on trackability is primarily on intruder detection and on algorithmic studies. A notable exception is [4], where the notion of detectability, the probability that an object on a path $L$ is detected, is discussed and some asymptotic results are given. Algorithmic results have been described in [1], [9], [11] to identify the best and worst sensed paths in a network when the sensor locations and the sensing radii are known. Statistical results via simulation are also presented in these papers. In [12], the same properties for the case of a network with a single sensor are studied. Further, [13] obtains the probability of detecting a target and uses this to develop a sequential deployment 


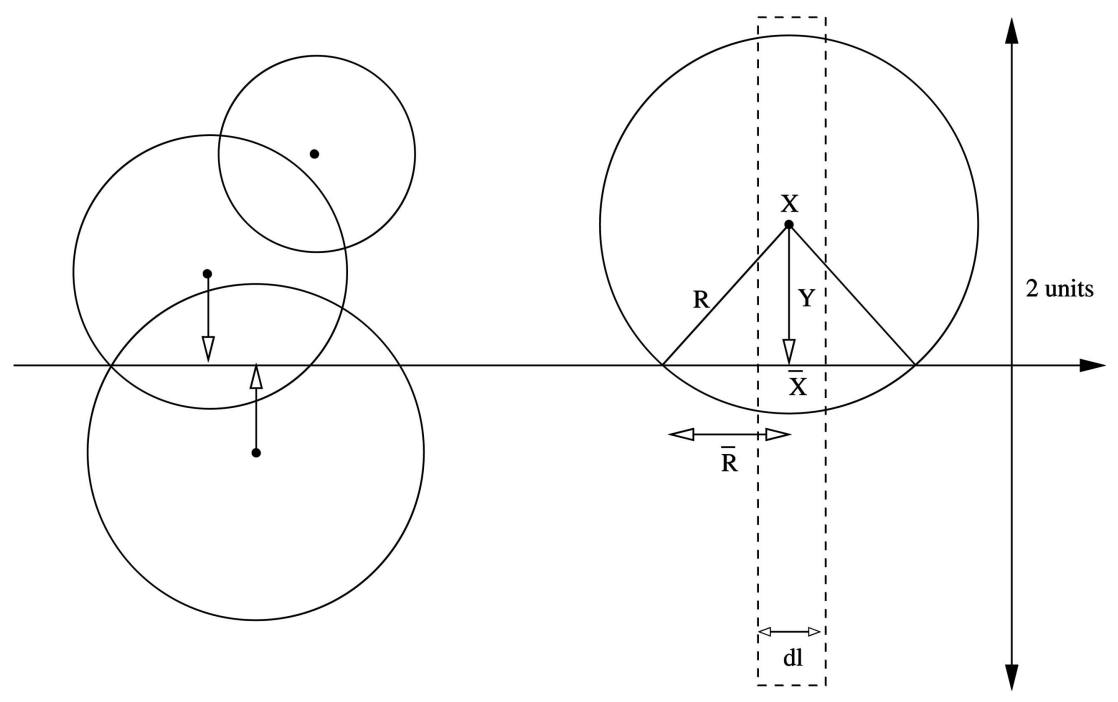

Fig. 2. The figure shows the projection of a sensor onto $\mathcal{L}$. The dots represent the sensors and the circles their sensing area. The region enclosed by vertical dotted lines is the differential region $d l$.

strategy to meet a QoS defined by the false alarm probability. Note that the detectability of [4] is the same as the exposure of [13]. Coverage, while maintaining connectivity, is studied in [14], [15]. In [14], it is proven that if the radio-range is at least twice the sensing range, complete coverage implies connectivity and, in [14], [15], algorithms are proposed to schedule sleep intervals in large-scale networks while meeting the required degree of coverage and connectivity requirements.

\section{Sensing Process on a Straight Line Path}

Let $L \in \Omega$ be an arbitrary straight line path of finite length. Let $\mathcal{L}$ be the line obtained by extending $L$ in both directions. Since the Boolean process is shift and rotation invariant (since the random sets $C_{i}$ are circles), without loss of generality, we can take $\mathcal{L}$ to be the $X$-axis of the coordinate axes. Since $R_{i}$ has a support of $[0,1]$, only sensors within a perpendicular distance of 1 from $\mathcal{L}$ may sense any part of $L$ and are of interest to us.

Construct a point process on $\mathcal{L}$ as follows: Mark all sensors that track some part of $\mathcal{L}$, i.e., mark a sensor if and only if its perpendicular distance to $\mathcal{L}$ is less than its sensing radius $R_{i}$. Project all the marked sensors onto $\mathcal{L}$ along the perpendicular to it. Denote the resulting point process on $\mathcal{L}$ by $\bar{F}$. For convenience, we will refer to the points of $\bar{F}$ as arrivals.

Lemma 1. $\bar{F}$ is a Poisson point process on $\mathcal{L}$ with rate $\bar{\lambda}=2 \lambda \beta$.

Proof. We prove the lemma by showing that the probability of an arrival in any differential length $d l$ of $\mathcal{L}$ is $\bar{\lambda} d l+o(d l)$ and that the arrivals have the independent increment property.

For any $l$, there is an arrival of $\bar{F}$ in $[l-d l / 2, l+d l / 2]$ if there is a marked sensor in the differential strip, $d P$, of thickness $d l$. Since the sensing radius has support in $[0,1]$, the length of the strip over which a marked sensor could be present is within 1 unit on either side of
$\mathcal{L}$. We restrict $d P$ to this range. This strip is centered at $l \in \mathcal{L}$. This is shown in Fig. 2 .

A sensor being present in $d P$ and it being marked are independent events. Thus, the probability that there is a marked sensor in $d P$ is the product of the probability of there being a sensor in $d P,(\lambda 2 d l+o(d l))$, and the probability that this is marked. We obtain this latter probability next.

If there is a sensor (say, $s$ with sensing radius $R$ ) in $d P$, then from the Poisson distribution of the sensors, its location is uniformly distributed in $d P$. This implies that the perpendicular distance of the sensor in $d P$ to $\mathcal{L}$, say, $Y$, will be uniformly distributed in $[0,1]$. For $s$ to be marked, its $R$ must be greater than $Y$. Therefore,

$$
\begin{aligned}
\operatorname{Pr}(s \text { is marked }) & =\int_{0}^{1} \operatorname{Pr}(Y \leq r) f_{R}(r) d r \\
& =\int_{0}^{1} r f_{R}(r) d r=\beta
\end{aligned}
$$

and

$$
\operatorname{Pr}\left(\text { Arrival in }\left[l-\frac{d l}{2}, l+\frac{d l}{2}\right]\right)=2 \lambda \beta d l+o(d l) .
$$

To prove the independent increment property, consider two nonoverlapping segments on $\mathcal{L}, L_{1}$, and $L_{2}$. Arrivals of $\bar{F}$ in $L_{1}$ and $L_{2}$ are decided by the presence of marked sensors in the rectangular regions $B_{1}$ and $B_{2}$ of height 2 and width equal to the width of $L_{1}$ and $L_{2}$ and centered at $L_{1}$ and $L_{2}$. Clearly, $B_{1}$ and $B_{2}$ are nonoverlapping and, by the independent increment property of the Poisson process of the sensor deployment, the point process $\bar{F}$ has the independent increment property.

Consider an arbitrary marked sensor $s$ located at $X$ and having sensing radius $R$. Let $Y$ be the perpendicular distance of $s$ to $\mathcal{L}$ and $\bar{X}$ be the projection of $X$ on $\mathcal{L}$. Note that both $R, Y$ are random variables and $\bar{X} \in \bar{F}$. Recall that, for thresholded sensing, $s$ will sense all points that are 
within a distance of $R$ from it. This means that, on $\mathcal{L}, s$ will sense the segment $[\bar{X}-\bar{R}, \bar{X}+\bar{R}]$, where $\bar{R}=\sqrt{R^{2}-Y^{2}}$. See Fig. 2.

Lemma 2. $\bar{R}$ is independent of $\bar{X}$ and its density, $f_{\bar{R}}(\bar{r})$, is

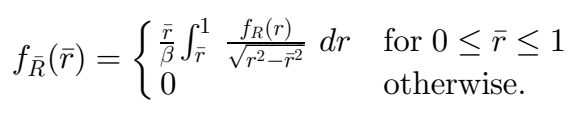

Proof. Since sensor $s$ is marked, $R \geq Y$. As discussed above, the segment on $\mathcal{L}$ sensed by $s$ is $[\bar{X}-\bar{R}, \bar{X}+\bar{R}]$, where $\bar{R}=\sqrt{R^{2}-Y^{2}}$. Since the sensor nodes are distributed as a homogeneous Poisson process, $Y$ is independent of $\bar{X}$. Further, $R$ is independent of $X$ and it follows that $\bar{R}$ is independent of $\bar{X}$. By a simple transformation of random variables, the distribution function of $\bar{R}, F_{\bar{R}}(\bar{r})$, can be written as

$$
F_{\bar{R}}(\bar{r})=\int_{r, y: \sqrt{r^{2}-y^{2} \leq \bar{r}}} f_{\{R, Y \mid R \geq Y\}}(r, y) d r d y .
$$

Here, $f_{R, Y}(\cdot, \cdot)$ is the joint density of $R$ and $Y$. The joint density conditioned on the event that the sensor is marked can be written as

$$
f_{\{R, Y \mid R \geq Y\}}(r, y)=\frac{f_{R, Y}(r, y)}{\operatorname{Pr}(R \geq Y)} .
$$

Recall from the proof of Lemma 1 that the probability that a sensor within a distance of 1 from $\mathcal{L}$ is marked is $\beta$. Further, as discussed before, $Y$ is uniformly distributed in $[0,1]$ and is independent of $R$. Therefore,

$$
f_{(R, Y \mid R \geq Y)}(r, y)=\frac{f_{R}(r)}{\beta} .
$$

Substituting for $f_{(R, Y \mid R \geq Y)}$ in (1) and then differentiating with respect to $\bar{r}$, we get the density function of $\bar{R}$ as

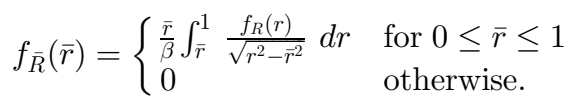

The "regions" on $\mathcal{L}$ that are sensed are the collection of segments $\left\{\bar{X}_{i}+\bar{I}_{i}\right\}$, where $\left\{\bar{X}_{i}\right\}$ is a Poisson process, $I_{i}$ is the random interval $\left[-\bar{R}_{i}, \bar{R}_{i}\right]$, and $\bar{R}_{i}$ s are i.i.d. random variables. Therefore, the sensing process on $\mathcal{L}$ is a onedimensional Boolean process. This means that the trackability of any straight line path can be studied as the coverage of a straight line of equal length by an appropriately defined one-dimensional Boolean process. It is easy to see that the latter is just an $M / G / \infty$ queue where the projected sensors are akin to the customer arrivals and the sensed segment of the path is the corresponding service time. There is one difference though. For the one-dimensional Boolean process described above, the centers of the sensing intervals are derived from a Poisson process, whereas, in the $M / G / \infty$ queue, the left endpoints of the service period form a Poisson process. Fortunately, from the discussion in [8, p. 80], there is a statistical equivalence between the two processes and the following can be shown.
Lemma 3. Consider a one-dimensional Boolean process $\left\{X_{i}+C_{i}\right\}$, where $\left\{X_{i}\right\}$ is a Poisson process, $C_{i}$ is the random interval $\left[-T_{i}, T_{i}\right]$, and the $T_{i} s$ are i.i.d. positive random variables. Then, $\left\{X_{i}+C_{i}\right\}$ has the same laws as the onedimensional Boolean process $\left\{X_{i}+C_{i}^{\prime}\right\}$, where $C_{i}^{\prime}$ is the random interval $\left[0,2 T_{i}\right]$.

The above discussion now leads us to state the key theorem of this paper:

Theorem 1. For thresholded sensing, the projected point process and the collection of sensed segments form a one-dimensional Boolean process with laws identical to the one-dimensional Boolean process $\left\{\bar{X}_{i}+\bar{C}_{i}\right\}$, where $\left\{\bar{X}_{i}\right\}$ is a Poisson point process of density $\bar{\lambda}=2 \lambda \beta, \bar{C}_{i}$ is the random interval $\left[0,2 \bar{R}_{i}\right]$, and the $\bar{R}_{i}$ s are i.i.d. random variables with density as in (2).

From the $\mathrm{M} / \mathrm{G} / \infty$ analogy, the theorem also says that the sensing process on $\mathcal{L}$ is statistically equivalent to an $\mathrm{M} / \mathrm{G} / \infty$ queue with arrival rate $\bar{\lambda}=2 \lambda \beta$ and service time density given by

$$
g(x)= \begin{cases}\frac{x}{4 \beta} \int_{\frac{x}{2}}^{1} \frac{f_{R}(r)}{\sqrt{r^{2}-\frac{x^{2}}{4}}} d r & \text { for } 0 \leq x \leq 2 \\ 0 & \text { otherwise }\end{cases}
$$

It can be shown that $\mathrm{E}(\bar{R})=\frac{\pi \mathrm{E}\left(R^{2}\right)}{4 \mathrm{E}(R)}$. For the special case when the sensing radii are degenerate with $R \equiv 1$, $f_{\bar{R}}(\bar{r})=\frac{\bar{r}}{\sqrt{1-\bar{r}^{2}}}$ for $0 \leq \bar{r} \leq 1$ and 0 outside. Further, $\beta=1$ and $\mathrm{E}(\bar{R})=\frac{\pi}{4}$.

\section{Exposure-Fraction Sensed}

In this section, we derive the critical sensor density that is required to $k$-sense a fraction $\alpha_{k}$ of a path $L$. We first look for asymptotic results and obtain a strong law for $k$-sensing and a central limit theorem for $k=1$. For finite networks, we obtain the expected fraction $k$-sensed and also the variance for $k=1$. Finally, through simulations, we investigate the robustness of the results to mild deviations from the system model.

\subsection{Asymptotic Analysis}

From the previous section, the event that a fraction $\alpha$ of the line segment is sensed by exactly $k$ sensors corresponds to the event that, in an $M / G / \infty$ queue, for a fraction $\alpha$ of an observation period of duration $l_{0}$, there are exactly $k$ customers in the system. From the ergodicity of the $\mathrm{M} / \mathrm{G} / \infty$ queue, as $l_{0} \rightarrow \infty$, this probability has a Poisson distribution with mean $\mu:=\bar{\lambda} 2 \mathrm{E}(\bar{R})=\pi \lambda \mathrm{E}\left(R^{2}\right)$. Therefore, for a straight line path $L$ of length $l_{0}$, as $l_{0} \rightarrow \infty$, the limiting fraction of a path that will be $k$-sensed is $\sum_{i=k}^{\infty} \frac{\mu^{i} e^{-\mu}}{i !}$.

We now obtain asymptotic results for finite length paths with increasing density of sensor nodes and decreasing sensing radii. The sensing radii are scaled by $\delta$ (i.e., the sensing radii are distributed as $\delta R$ ) such that $\delta^{2} \lambda \rightarrow \rho$, $0 \leq \rho<\infty$, as $\lambda \rightarrow \infty$. This scaling of the sensing radii allows us to derive sensing properties in the large density limit. With this scaling, the sensing statistics of the finite length segment $L$ will be the same as the limiting statistics 
of $L$ as $l_{0} \rightarrow \infty$ in a network where $\delta=1$ and the sensor density is fixed at $\rho$. This follows from the discussion on the scaling properties of Boolean processes in [8]. From this, we can state the following strong law:

Theorem 2. Let $\bar{\rho}=\pi \rho \mathrm{E}\left(R^{2}\right)$. Let $\delta \rightarrow 0$ and $\lambda \rightarrow \infty$ such that $\delta^{2} \lambda \rightarrow \rho, 0 \leq \rho<\infty$. Then, with probability 1,

$$
\alpha_{k}:=\mathrm{X}_{\psi_{T}(x, k)}(L) \rightarrow \sum_{i=k}^{\infty} \frac{\bar{\rho}^{i} e^{-\bar{\rho}}}{i !} .
$$

The limiting value of $\alpha_{1}$ for any path is $\left(1-e^{-\pi \rho \mathrm{E}\left(R^{2}\right)}\right)$ with probability 1. From Theorem 3.6 in [8], this is also the fraction of any finite area that is almost surely 1-covered in the same limiting regime! In fact, from Theorem 2 of [5], this equality also extends to $k>1$. While this seems reasonable, it is not obvious that this should be the case.

We next obtain a central limit theorem for 1-sensing to complement the almost sure convergence in Theorem 2 . On scaling the sensing radii by $\delta$, it is easy to see that the density of the induced coverage process on $\mathcal{L}, \bar{\lambda}$, is also scaled by $\delta$. Therefore, $\bar{\lambda}=2 \lambda \beta \delta$. Therefore, scaling $R$ by $\delta$ such that $\lambda^{2} \delta \rightarrow \rho$ as $\lambda \rightarrow \infty$ and $\delta \rightarrow 0$ implies that $\delta \bar{\lambda} \rightarrow 2 \beta \rho$. From Theorem 3.5 in [8], where a central limit theorem for the length of the path that is not 1-sensed is derived, we can obtain a central limit theorem for $\mathrm{X}_{\psi_{T}(x, k)}(L)$.

Theorem 3. If $\delta \rightarrow 0$ as $\lambda \rightarrow \infty$ such that $\delta^{2} \lambda \rightarrow \frac{-\ln (1-\alpha)}{\pi \mathrm{E}\left(R^{2}\right)}$, then

$$
\sqrt{2 \beta \delta \lambda}\left(\mathrm{X}_{\psi_{T}(x, 1)}(L)-\alpha\right) \quad \rightarrow \quad N\left(0, \sigma^{2}\right),
$$

where

$$
\begin{aligned}
& \sigma^{2}=\frac{4 \beta \ln (1-\alpha)}{\pi \mathrm{E}\left(R^{2}\right)(1-\alpha)^{2} l_{0}} \times \\
& \int_{0}^{\infty}\left(\exp \left(\frac{-2 \beta \ln (1-\alpha)}{\pi \mathrm{E}\left(R^{2}\right)} \int_{x}^{\infty}\left(1-F_{\bar{R}}(y)\right) d y\right)-1\right) d x
\end{aligned}
$$

and $F_{\bar{R}}(\cdot)$ is the distribution function of $\bar{R}$ from Theorem 1 .

We make two observations: The speed of convergence of the asymptotics is of the order of $\lambda^{\frac{1}{2}}$. Though the asymptotic mean for a two-dimensional area coverage and one-dimensional path coverage are the same, the normalized variances, $\sigma^{2}$, in (4) and in Theorem 3.5 of [8] are not equal.

\subsection{Finite Network Analysis}

For finite $\lambda$, we can obtain the expectation and variance of $\mathrm{X}_{\psi_{T}(x, 1)}(L)$ by a simple application of the results of Section 3.2 of [8]:

$$
\begin{aligned}
& \mathrm{E}\left(\mathrm{X}_{\psi_{T}(x, 1)}\right)(L)=1-e^{-\lambda \pi \mathrm{E}\left(R^{2}\right)} \\
& \operatorname{VAR}\left(\mathrm{X}_{\psi_{T}(x, 1)}\right)(L)=\frac{2 e^{-2 \pi \lambda \mathrm{E}\left(R^{2}\right)}}{l_{0}^{2}} \times \\
& \int_{0}^{l_{0}}\left(l_{0}-x\right)\left(e^{2 \beta \lambda \int_{x}^{\infty}\left(1-F_{\bar{R}}(y)\right) d y}-1\right) d x .
\end{aligned}
$$

Observe that $\mathrm{E}\left(\mathrm{X}_{\psi_{T}(x, 1)}\right)(L)$ has the same form as the asymptotic $\mathrm{E}\left(\mathrm{X}_{\psi_{T}(x, 1)}\right)(L)$ value except for $\rho$ being replaced by $\lambda$. We plot $\operatorname{VAR}\left(\mathrm{X}_{\psi_{T}(x, 1)}\right)(L)$ as a function of $l_{0}$ for different $\lambda$ in Fig. 3. Observe that, even for small values of $\lambda$

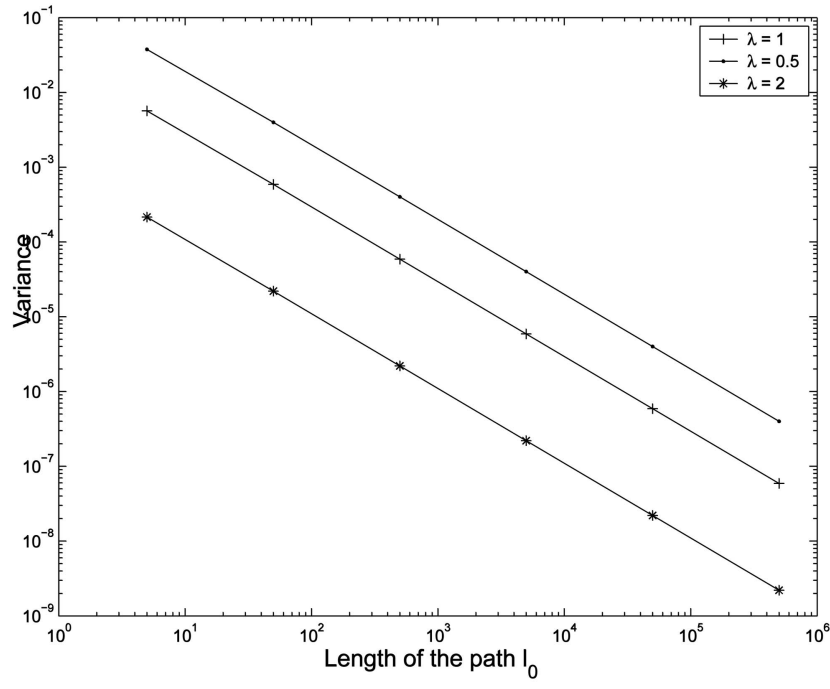

Fig. 3. The variance of $\mathrm{X}_{\psi_{T}(x, 1)}(L)$ as a function of $l_{0}$, the length of the path for a finite lambda for $R \equiv 1$.

and $l_{0}$, the variance is small compared to the mean. Thus, in a realization of a network, the fraction of a path $L$ that will be 1-sensed will not be very different from the mean. Thus, to 1-sense a fraction $\alpha$ of a path $L$, the sensor density must be $\lambda=1-e^{-\pi \rho \mathrm{E}\left(R^{2}\right)}$.

For a general $k$, from the queuing analogy and the ergodicity of the $M / G / \infty$ queue, it follows that the expected fraction of a path $L$ that will be $k$-sensed in a network of density $\lambda$ is

$$
\mathrm{E}\left(X_{\psi_{T}(x, k)}\right)=\sum_{i=k}^{\infty} \frac{\left(\pi \mathrm{E}\left(R^{2}\right) \lambda\right)^{i} e^{-\pi \mathrm{E}\left(R^{2}\right) \lambda}}{i !} .
$$

However, a closed form expression for the variance of $X_{\psi_{T}(x, k)}$ is not available and we cannot conclude, as we did for $k=1$, that the fraction of $L$ that will be $k$-sensed in a realization of a network will be close to the expected value. We therefore perform simulations to evaluate the variance. We simulate 10,000 network deployments and obtain the fraction of the path $L$ that is $k$-sensed. We fix $\lambda=0.5$. For $k=1,2,3,4,5$, the expected fraction of $L$ that will be $k$-sensed can be obtained from (5) to be 0.4076, 0.0974, $0.0162,0.0021$, and 0.0002 , respectively. Fig. 4 plots the sample variance of the fraction of $L$ that was $k$-sensed as a function of $l_{0}$ for $k=1,2,3,4,5$. We see that the variance is less than 5 percent when $l_{0}=10$ and further decreases exponentially with $l_{0}$. A similar behavior has been observed for other values of $\lambda$. We can therefore conclude that, in a network realization, the fraction of a path $L$ that will be $k$-sensed will be close to the expected value. Therefore, (5) can be solved to obtain $\lambda_{c}\left(\alpha_{k}\right)$, the minimum $\lambda$ required to sense at least a fraction $\alpha(0<\alpha \leq 1)$ of $L . \lambda_{c}\left(\alpha_{k}\right)$ can be written as

$$
\lambda_{c}\left(\alpha_{k}\right)=\frac{\bar{\lambda}_{c}\left(\alpha_{k}\right)}{\pi \mathrm{E}\left(R^{2}\right)},
$$




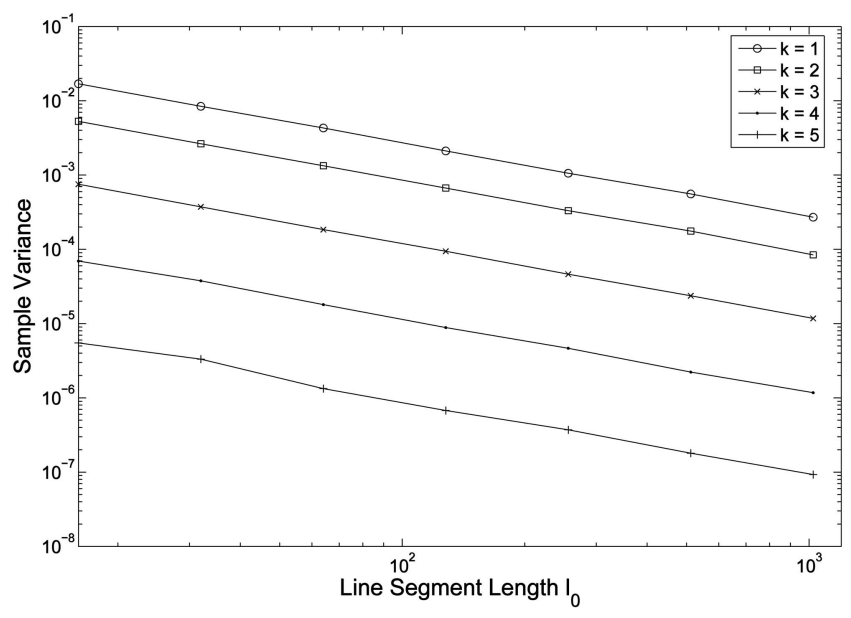

Fig. 4. Sample variance against $l_{0}$ for $k=1,2,3,4,5$ and $\lambda=0.5$.

where $\bar{\lambda}_{c}\left(\alpha_{k}\right)$ is the solution to (3). Equation (3) can be explicitly solved to obtain a closed form for $\bar{\lambda}_{c}\left(\alpha_{k}\right)$ only for $k=1$. Doing this, we get

$$
\lambda_{c}\left(\alpha_{k}\right)=\frac{-\ln (1-\alpha)}{\pi \mathrm{E}\left(R^{2}\right)} .
$$

For $2 \leq k \leq 6$, (3) is numerically solved to obtain $\bar{\lambda}_{c}\left(\alpha_{k}\right)$. A plot of $\bar{\lambda}_{c}\left(\alpha_{k}\right)$ as a function of $\alpha$ is plotted in Fig. 5. For these $k$, we observe that $\bar{\lambda}_{c}\left(\alpha_{k}\right)$ is approximately proportional to $\alpha$ for $0.1 \leq \alpha \leq 0.8$. When $\alpha$ is close to one (complete tracking), any incremental increase in the requirement of $\alpha$ will result in a steep increase in the requirement of $\lambda$. Also, for a given $\alpha, \bar{\lambda}_{c}\left(\alpha_{k}\right)$ is also proportional to $k$.

Let us now investigate the increase in $\bar{\lambda}_{c}\left(\alpha_{k}\right), \Delta \bar{\lambda}_{c}\left(\alpha_{k}\right)$, that is required to achieve a given increase in $\alpha_{k}$. Fig. 6 shows $\Delta \bar{\lambda}_{c}\left(\alpha_{k}\right)$ as a function of $\alpha$ for different $k$ to increase the fraction of the path sensed from $\alpha$ to $\alpha+0.05$. While the plot for $k=1$ is strictly increasing, the plots for $k>1$ have a minimum. The reason for this is as follows: For a given $\bar{\lambda}$, from the stationary distribution of the $\mathrm{M} / \mathrm{G} / \infty$ queue, the difference between the fraction of $L$ that is $k$-sensed and the fraction that is $(k+1)$-sensed is $e^{-\bar{\lambda} \frac{\lambda^{k}}{k !}}$, which is just the

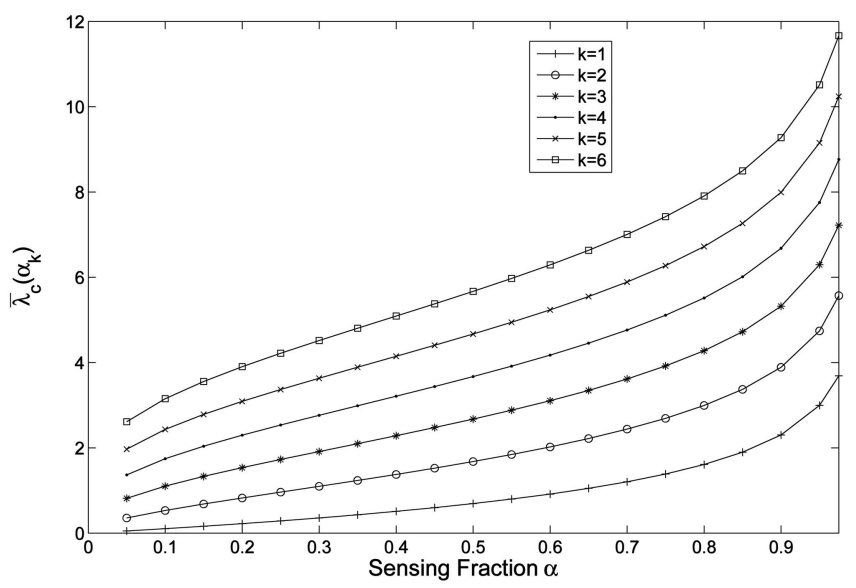

Fig. 5. The figure shows the plot of $\bar{\lambda}_{c}\left(\alpha_{k}\right)$ with $\alpha$ for $1 \leq k \leq 6$. $\lambda_{c}\left(\alpha_{k}\right)=\frac{\bar{\lambda}_{c}\left(\alpha_{k}\right)}{\pi \mathrm{E} R^{2}}$.

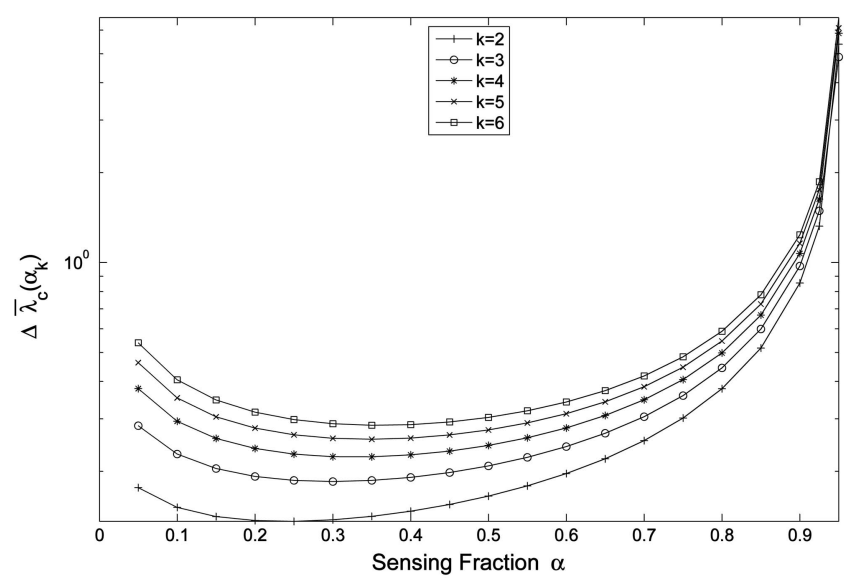

Fig. 6. Plot of $\Delta \bar{\lambda}_{c}\left(\alpha_{k}\right)$ as a function of $\alpha$ for different $k . \Delta \bar{\lambda}_{c}\left(\alpha_{k}\right)$ is the increase in $\bar{\lambda}_{c}\left(\alpha_{k}\right)$ that is required to increase the fraction of a line that is sensed by 5 percent, i.e., the increase in $\bar{\lambda}_{c}\left(\alpha_{k}\right)$ required to increase the fraction sensed from $\alpha$ to $\alpha+0.05$.

fraction of the line that is sensed by exactly $k$ sensors. This difference has a maximum at $\bar{\lambda}=k$. This implies that, for low values of $\bar{\lambda}$, increasing the sensor density by a fixed amount will convert a larger portion of the area from being $k$-sensed to becoming $(k+1)$-sensed up to $\bar{\lambda}=k$, after which, the additional fraction added decreases.

\subsection{Robustness to the Model Assumptions}

We analyze the robustness of the results derived above when the model assumptions do not hold. Specifically, we consider two departures from the assumptions-curvilinear paths and nonhomogeneous sensor density.

\subsubsection{Curvilinear Paths}

The asymptotic results derived for straight line paths also extend to piecewise linear and curvilinear paths. This can be seen as follows: As $\delta \rightarrow 0$, a sensor can sense at most one segment of a piecewise linear path. Hence, the sensing properties of each linear segment of the path are independent and can be analyzed separately. A curvilinear path can be approximated by piecewise linear segments. Thus, the asymptotic results obtained for straight line paths extend to curvilinear paths also.

We now consider finite networks and curvilinear paths which are arcs of circles of different radii, denoted by $R_{c}$, over a chord of length 100. These paths are shown in Fig. 7. We obtain the fraction that is $k$-sensed for and compare it with $\mathrm{E}\left(X_{\psi_{T}(x, k)}\right)$ derived in (5) for straight line paths. We fix $\lambda=0.5$ and $R \equiv 1$. The results are summarized in Table 1 . We see that the mean fraction of the curvilinear paths that is

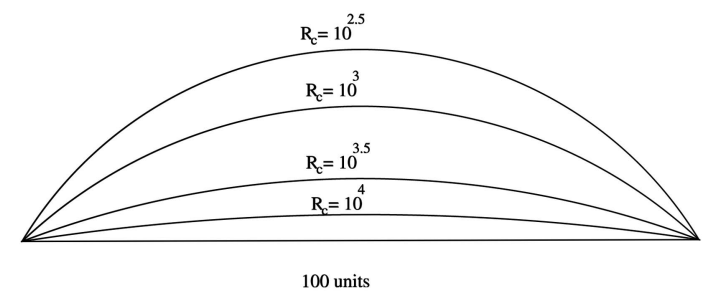

Fig. 7. Paths of curvature $R_{c}=10^{2.5}, 10^{3}, 10^{3.5}, 10^{4}$. The sensor density is fixed at $\lambda=0.5$ and $R \equiv 1$. 
TABLE 1

Sample Mean of the Fraction of Curvilinear Paths That Was $k$-Sensed for Different $R_{c}$

\begin{tabular}{|l|l|l|l|l|l|l|l|l|l|l|}
\hline$R_{c}$ & \multicolumn{2}{|c|}{$k=1$} & \multicolumn{2}{c|}{$k=2$} & \multicolumn{2}{c|}{$k=3$} & \multicolumn{2}{c|}{$k=4$} & \multicolumn{2}{c|}{$k=5$} \\
\hline & Mean & MSD & Mean & MSD & Mean & MSD & Mean & MSD & Mean & MSD \\
\hline $10^{2.5}$ & 0.7916 & 0.0039 & 0.4655 & 0.0071 & 0.2091 & 0.0045 & 0.0752 & 0.0016 & 0.0222 & 0.0004 \\
$10^{3}$ & 0.7898 & 0.0041 & 0.4626 & 0.0063 & 0.2072 & 0.0043 & 0.0738 & 0.0016 & 0.0215 & 0.0004 \\
$10^{3.5}$ & 0.7910 & 0.0040 & 0.4646 & 0.0060 & 0.2083 & 0.0038 & 0.0741 & 0.0015 & 0.0219 & 0.0004 \\
$10^{4}$ & 0.7928 & 0.0040 & 0.4670 & 0.0067 & 0.2100 & 0.0042 & 0.0754 & 0.0015 & 0.0221 & 0.0004 \\
\hline$\infty$ & 0.7921 & - & 0.4656 & - & 0.2091 & - & 0.0748 & - & 0.0055 & \\
\hline
\end{tabular}

$R_{c}=\infty$ corresponds to the straight line. We also show the mean square deviation (MSD) from that of the straight line path.

$k$-sensed is very close to the fraction of straight line paths $k$-sensed. Further, the mean square deviation between the actual fraction of the curvilinear path that is $k$-sensed and the expected fraction of a straight line path that is $k$-sensed is less than 5 percent. Thus, $\lambda_{c}\left(\alpha_{k}\right)$ can be used as the critical density to $k$-sense a fraction of $\alpha_{k}$ of curvilinear paths.

\subsubsection{Nonhomogeneous Networks}

We next consider nonhomogeneous networks. We assume that the sensors form a spatial nonhomogeneous Poisson process with density $\lambda(x, y)=\theta e^{-\mu(|x|+|y|)}$. Through simulations, we obtain the fraction of a straight line path that is $k$-sensed. To obtain a comparable result for the homogeneous network, we use

$$
\lambda=\frac{1}{\|A\|} \int_{A} \lambda(x, y) d x d y
$$

in (5). Here, $A$ is the area around $L$ where the sensors can sense $L$ and $\|A\|$ is the area of $A$. The expected fraction sensed for the two cases are plotted in Fig. 8 as a function of $\mu$ for different $l_{0} . \theta$ is fixed at 0.5 and $R$ is assumed to be uniform in $[0,1]$. We see that the mean fraction of the path $k$-sensed in the nonhomogeneous network is close to the expected fraction $k$-sensed in the homogeneous network. As expected, as $\eta$ reduces, the deviation also reduces.

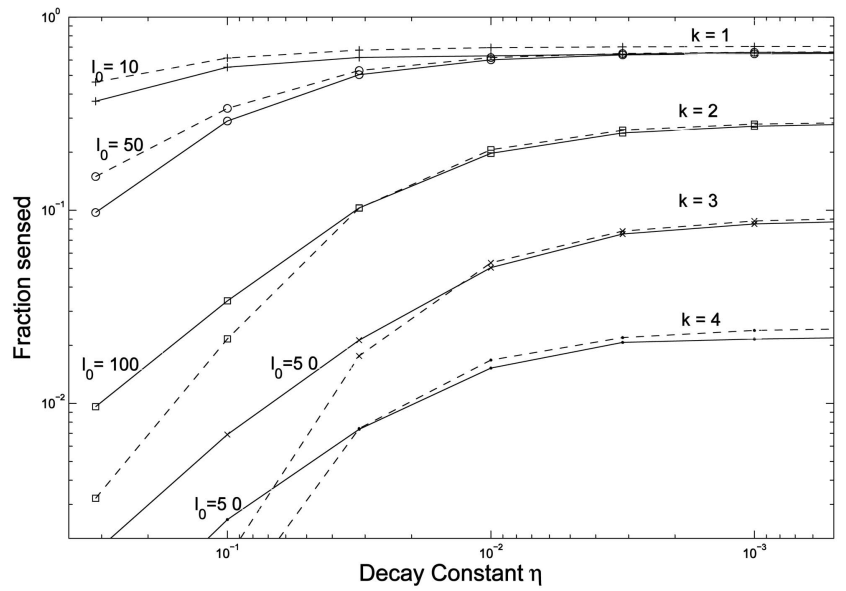

Fig. 8. Plot of fraction $k$-sensed against decay constant for different values of $l_{0}$ and $k$. The dashed line is the fraction sensed in a homogeneous network.

\section{1-Sensing: Length to FiRSt Sense AND SENSING Continuity}

Since the Boolean process is shift invariant, without loss of generality, $L$ can be taken to be the segment $\left[0, l_{0}\right]$. Also, in the following, we consider networks with finite $\lambda$.

\subsection{Length to First Sense}

Let the regions $x<0,0 \leq x<l_{0}$, and $l_{0} \leq x$ in $\Re^{2}$ be denoted by $W_{1}, W_{2}$, and $W_{3}$, respectively. Define the regions $W_{i}(d) i=1,2,3$ as follows: $W_{1}(d)$ is the rectangle with $(0, d)$ and $\left(l_{0},-d\right)$ as the opposite corners. $W_{2}(d)$ and $W_{3}(d)$ are the semicircular regions of radius $d$ with centers at $(0,0)$ and $\left(0, l_{0}\right)$, respectively. See Fig. 9 .

Let $E_{u}:=E_{1} \cap E_{2} \cap E_{3}$, where $E_{i}, i=1,2,3$, is the event that no part of $L$ is 1 -sensed by any sensor in $W_{i}$. Let $E_{d}$ be the complement of $E_{u}$, i.e., the event that $L$ is 1 -sensed. Since the $W_{i}$ s are nonoverlapping, the $E_{i}$ s are independent and $\operatorname{Pr}\left(E_{u}\right)$ is the product of the probabilities of the $E_{i}$. We calculate these probabilities next.

Since the sensing radius has support in $[0,1]$, only sensors in $W_{i}(1)$ can sense any part of $L$. Further, since the sensor locations form a spatial Poisson process of density $\lambda$, the number of sensors in $W_{1}(1), N_{1}$, will be a Poisson random variable with mean $2 l_{0} \lambda$ and, from the proof of

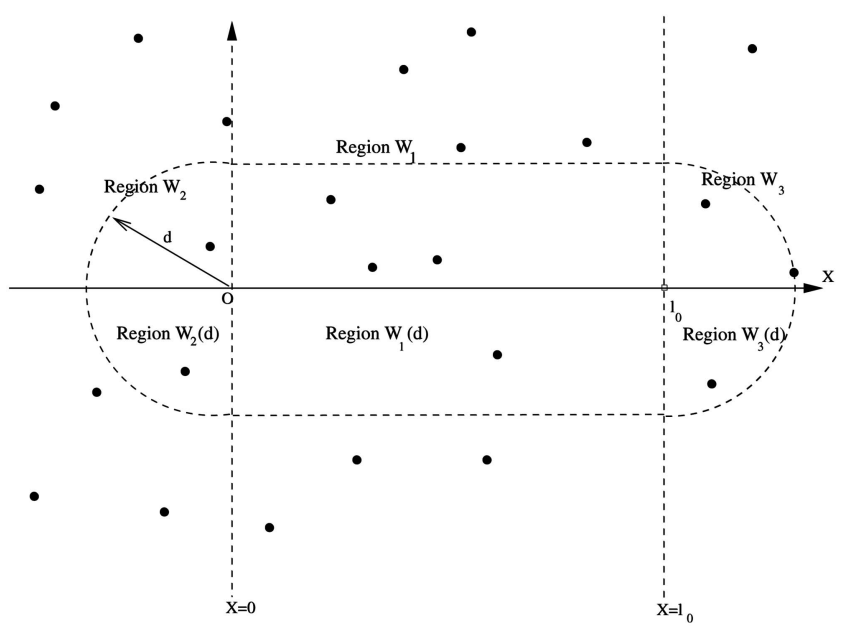

Fig. 9. The dotted lines mark the boundary of the regions $W_{1}(d), W_{2}(d)$, and $W_{3}(d)$. The two lines $x=0$ and $x=l_{0}$ also mark the boundary of $W_{1}, W_{2}$, and $W_{3}$. 


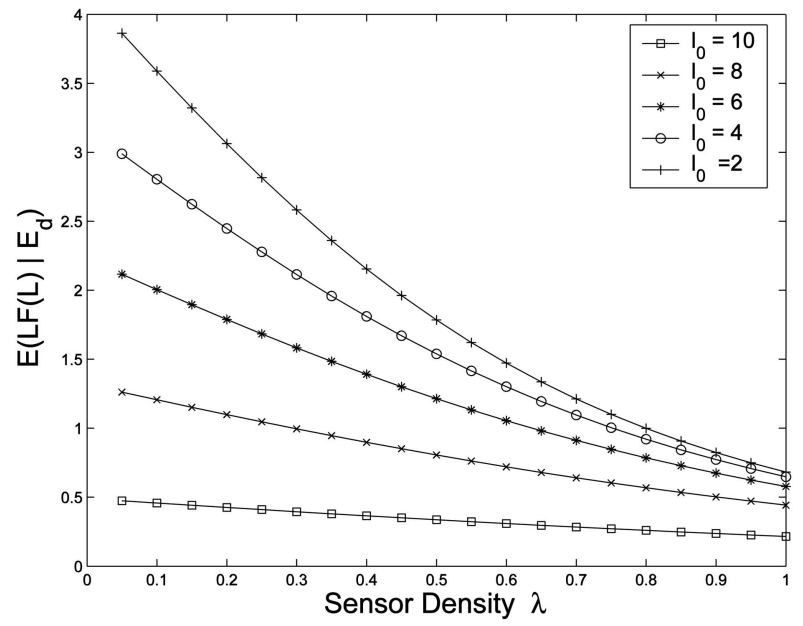

Fig. 10. The conditional expectation of $L F(L)$ given that the path is sensed is plotted as a function of $\lambda$ for different values of $l_{0}$.

Lemma 1 , the probability that any of these sensors will sense $L$ is $\beta$. Therefore,

$$
\operatorname{Pr}\left(E_{1}\right)=\mathrm{E}\left((1-\beta)^{N_{1}}\right)=e^{-2 l_{0} \lambda \beta} .
$$

From symmetry, the probabilities of $E_{2}$ and $E_{3}$ are equal and we evaluate the probability of $E_{2}$. Given that a sensor is in $W_{2}(1)$, its location is uniformly distributed in that region. Hence, the probability that this sensor does not sense $(0,0)$ is

$$
\frac{1}{2} \int_{0}^{1} f_{R}(r)\left(1-r^{2}\right) d r=\frac{1-\mathrm{E}\left(R^{2}\right)}{2} .
$$

$N_{2}$, the number of sensors in $W_{2}(1)$, is a Poisson random variable with mean $\lambda \frac{\pi}{2}$. Therefore,

$$
\operatorname{Pr}\left(E_{2}\right)=\mathrm{E}\left(\left(1-\mathrm{E}\left(R^{2}\right)\right)^{N_{2}}\right)=e^{-\frac{\pi}{2} \lambda \mathrm{E}\left(R^{2}\right)} .
$$

Therefore, it follows that

$$
\operatorname{Pr}\left(E_{d}\right)=1-e^{-\lambda\left(\pi \mathrm{E}\left(R^{2}\right)+l_{0} \mathrm{E}(R)\right)} .
$$

We use this to obtain the distribution of $L F(L), F_{L F(L)}(x)$. Clearly, $\operatorname{Pr}(L F(L)<x)$ is 0 for $x<0$ and 1 for $x>l_{0}$. For $x \in\left[0, l_{0}\right), F_{L F(L)}(x)$ is the probability that $[0, x)$ is sensed, which can be obtained from (6) by replacing $l_{0}$ by $x$. Therefore,

$$
F_{L F(L)}(x)= \begin{cases}0 & \text { if } x<0 \\ 1-e^{-\lambda \pi \mathrm{E}\left(R^{2}\right)-\lambda \mathrm{E}(R) x} & \text { if } 0 \leq x<l_{0} \\ 1 & \text { if } x>l_{0} .\end{cases}
$$

From above, notice that the probability density of $L F(L)$ has point masses at 0 and $l_{0}$ corresponding to the probabilities of the beginning of the path being sensed and the path not being sensed at all. Observe that this is just the truncated exponential distribution.

In Figs. 10 and 11, we show the expectation and variance of $L F(L)$ conditioned on the path being sensed for the degenerate case of $R \equiv 1$. As expected $\mathrm{E}(L F(L))$ decreases with $\lambda$ for the case of $R \equiv 1$. The conditional expectation and variance of $L F(L)$ decreases exponentially with $\lambda$. Observe that, even for moderate $\lambda$, the conditional expectation and the variance of $L F(L)$ do not significantly depend

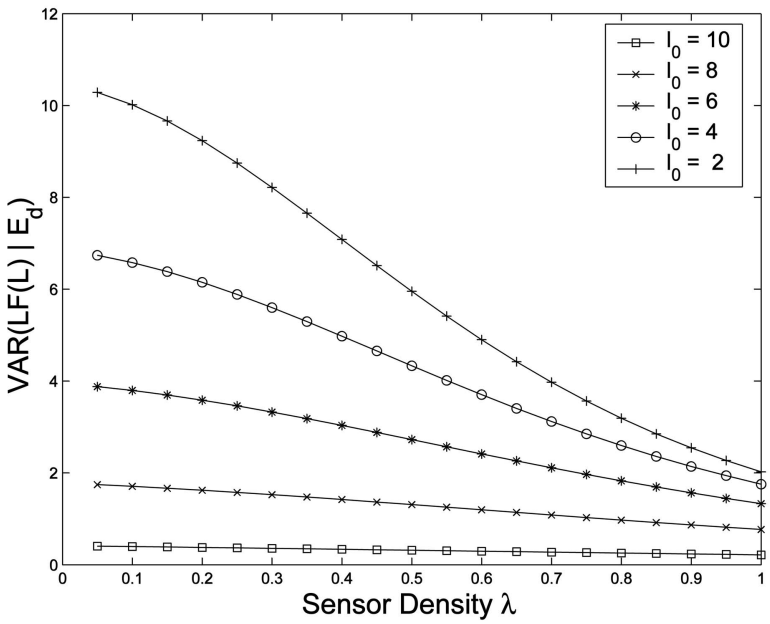

Fig. 11. The conditional variance of $L F(L)$ given that the path is sensed is plotted as a function of $\lambda$ for different values of $l_{0}$.

on $l_{0}$. Further, for large $l_{0}$, the dependence on $\lambda$ is also not very strong.

\subsection{Sensing Continuity: Clumps and Holes}

Since the sensing process on the path is a one-dimensional Boolean process, for 1-sensing, the hole lengths are clearly exponentially distributed with rate $\bar{\lambda}$, i.e., the the hole length density is $f_{H}(x)=\bar{\lambda} e^{-\bar{\lambda} x}$. From Theorem 2.2 of [8], the characteristic function, $\gamma_{Z}(s)$, of length of a clump, $Z$, is

$\gamma_{Z}(s)=\frac{\bar{\lambda}+s}{\bar{\lambda}}-\left(\bar{\lambda} \int_{0}^{\infty} \exp \left(-s t-\bar{\lambda} \int_{0}^{t}\left(1-F_{\bar{R}}(x)\right) d x\right) d t\right)^{-1}$.

The expectation of the clump length is $\mathrm{E}(Z)=\bar{\lambda}^{-1}\left(e^{2 \bar{\lambda} \mathrm{E}(\bar{R})}-\right.$ 1) and its variance is

$$
\begin{aligned}
& \operatorname{VAR}(Z)=-\left(\bar{\lambda}^{-2}\left(e^{2 \bar{\lambda} \mathrm{E}(\bar{R})}-1\right)^{2}\right)+ \\
& \quad+\frac{2 e^{2 \bar{\lambda} \mathrm{E}(\bar{R})}}{\bar{\lambda}} \int_{0}^{\infty}\left(\exp \left(\bar{\lambda} \int_{y}^{\infty}\left\{1-F_{\bar{R}}(x)\right\} d x\right)-1\right) d y .
\end{aligned}
$$

Fig. 12 is a log-plot of the variance of the length of a clump length as a function of $\lambda$. Observe that the plot is a straight line, which means that the $\operatorname{VAR}(Z)$ increases exponentially with $\lambda$. This means that, for high $\alpha$ and, hence, for high $\lambda$, i.e., in the high tracking regime, the durations for which the target would be tracked continuously varies a lot around the mean clump length even though the total proportions of time it is tracked may not vary a lot. This in turn implies that a target will encounter a large number of small clumps or a small number of large clumps. This implies that, in spite of ensuring high coverage, there is a lot of variability in the quality of tracking.

Theorems 2.3 and 2.4 [8, p. 141] can be used to obtain limiting distributions for the clump lengths.

Theorem 4. As $\lambda \rightarrow \infty$, the distribution of $\frac{Z}{\mathbf{E}(Z)}$ goes to an exponential with mean 1.

Further, observe that

$$
\log x \int_{x}^{\infty}\left\{1-F_{\bar{R}}(r)\right\} \rightarrow 0
$$




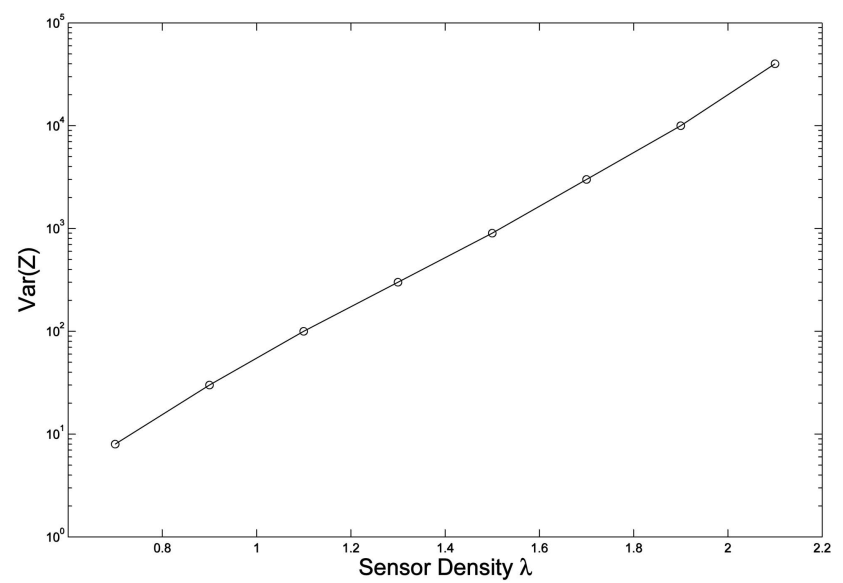

Fig. 12. The variance of $Z$, the clump length, is plotted as a function of $\lambda$.

as $x \rightarrow 0$. Here, $F_{\bar{R}}(r)$ is the distribution function of $\bar{R}$. Therefore, from Theorem 2.4 in [8], we have:

Theorem 5. If the sensing radii $R$ are scaled by $\delta, \delta \rightarrow 0$, and if $\lambda \rightarrow \infty$ such that

$$
4 \mathrm{E}(\bar{R}) \beta \lambda \delta^{2}=\ln \left(\frac{2 \beta \delta \lambda}{u}\right)+o(1)
$$

then, in the limit, the distribution of $Z$ goes to an exponential with mean $u$, a constant.

We next obtain asymptotic results for the number of holes and clumps in $L$. A one-dimensional Boolean process is essentially a renewal process on $\mathcal{L}$, with renewal cycle length, $D$, equal to $H+Z$. Since $H$ and $Z$ are independent,

$$
\mathrm{E}(D)=\mathrm{E}(H)+\mathrm{E}(Z)=\frac{e^{\pi \mathrm{E}\left(R^{2}\right) \lambda}}{2 \lambda \beta}
$$

and $\operatorname{VAR}(D)=\frac{1}{\lambda^{2}}+\operatorname{VAR}(Z)$. The number of holes, $N_{H}(L)$, and clumps, $N_{Z}(L)$, in $L$ will be equal to or 1 less than the number of renewals in $L$. Therefore, from the renewal theorem as $l_{0} \rightarrow \infty, \frac{N_{H}(L)}{l_{0}}$ and $\frac{N_{Z}(L)}{l_{0}}$ will converge to $2 \lambda \beta e^{-\pi \lambda \mathrm{E}\left(R^{2}\right)}$. This result, as in Section 4 , can be extended

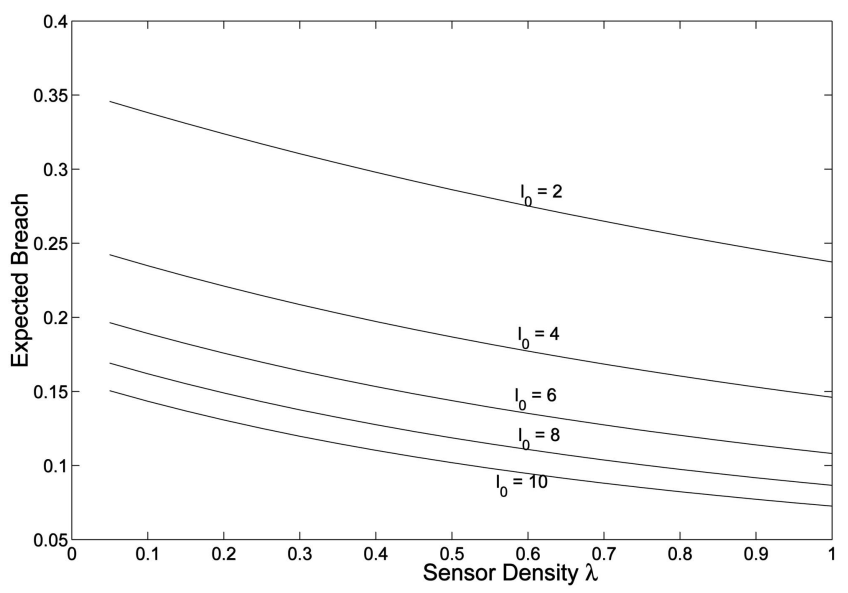

Fig. 13. Plot of $\mathrm{E}(\operatorname{Br}(L))$ against $\lambda$ for different values of $l_{0}$.

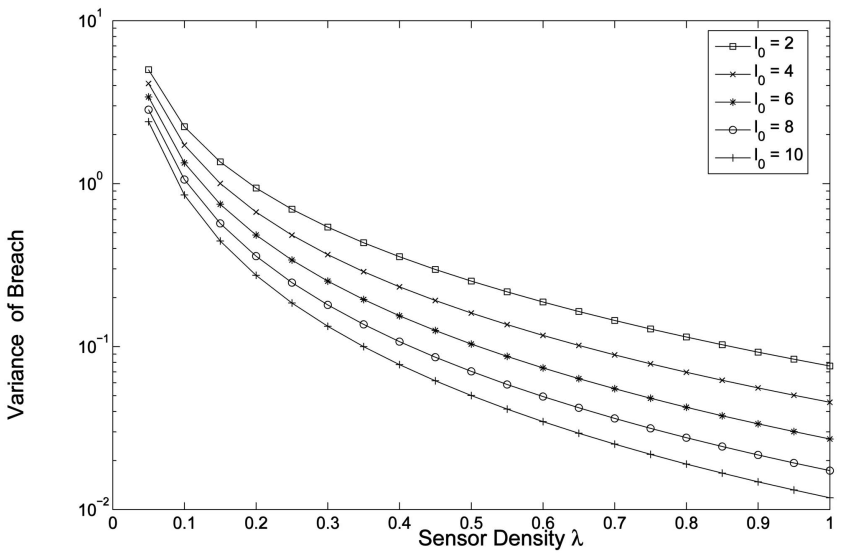

Fig. 14. Plot of $\operatorname{VAR}(B r(L))$ against $\lambda$ for different values of $l_{0}$.

to obtain asymptotics for $N_{H}(L)$ and $N_{Z}(L)$ when $l_{0}$ is finite by scaling $R$ to $\delta R$ such that $\delta \rightarrow 0$ and $\delta^{2} \lambda \rightarrow \rho$.

Theorem 6. If $\delta \rightarrow 0$ and $\lambda \rightarrow \infty$ such that $\delta^{2} \lambda \rightarrow \rho$, $0 \leq \rho<\infty$, then, with probability 1 ,

$$
\frac{N_{H}(L)}{l_{0}}=\frac{N_{Z}(L)}{l_{0}} \rightarrow 2 \rho \beta e^{-\pi \rho \mathrm{E}\left(R^{2}\right)} .
$$

Further, it is possible to derive a central limit theorem for $N_{H}$ and $N_{Z}$ from the central limit theorem for number of renewals. Additional statistics may be obtained by suitably using the results from $[8, \mathrm{ch} .4]$.

\section{BREACH AND SUPPORT}

Recall that breach is the distance of the closest sensor to $L$. Therefore, $F_{B r(L)}(d)$ is the probability that there is at least one sensor within a distance of $d$ from $L$. The latter is the probability that there is at least one sensor in the region $W_{i}(d) \cup W_{2}(d) \cup W_{3}(d)$, which is of area $2 l_{0} d+\pi d^{2}$ (see Fig. 9). Since the sensor locations form a spatial Poisson process, we get

$$
F_{B r(L)}(d)=1-e^{-\left(\lambda \pi d^{2}+\lambda l_{0} d\right)}
$$

Fig. 13 and Fig. 14 plots the expected value and variance of breach, respectively, as a function of $\lambda$ for different values of $l_{0}$. Observe the exponential decrease of both with $\lambda$. For a fixed $\lambda$, the relative change in the expected breach is higher for lower $l_{0}$, while this is not so pronounced for the variance. Further, the relative change in the variance with change in $l_{0}$ is higher for higher $\lambda$, which is not the case for the expectation.

To calculate the support, we first derive the distribution of $B_{i}$, the distance to $L$ of the closest sensor in $W_{i}$. Let $N_{i}(d)$ denote the number of sensors in $W_{i}(d)$ and $E_{i}(d)$ denote the event that $N_{i}(d) \neq 0$.

Observe that, in the limit as $d \rightarrow \infty$, the region $W_{i}(d)$ approaches $W_{i}$ and the probability of $E_{i}(d)$ approaches 1 . Thus, the density of $B_{i}$ can be obtained by first conditioning on $E_{i}(d)$ and then taking the limit $d \rightarrow \infty$. We use this strategy below to calculate the densities.

Given that there are sensors in $W_{1}(d)$, they will be uniformly distributed in $W_{1}(d)$. This means that the perpendicular distance of the sensors to $L$, which is also 
their shortest distance to $L$, has a uniform density in $[0, d]$. Therefore, conditioned on $E_{1}(d)$ and $N_{1}(d), B_{1}$ will be the minimum of $N_{1}(d)$ random variables that are independent and uniformly distributed in $[0, d]$.

$$
f_{B_{1} \mid E_{1}(d)}(x)= \begin{cases}\mathrm{E}\left(\frac{N_{1}(d)}{d}\left(1-\frac{x}{d}\right)^{N_{1}(d)-1}\right) & \text { if } x<d \\ 0 & \text { otherwise. }\end{cases}
$$

Further, conditioned on $E_{1}(d)$, the density of $N_{1}(d)$ is

$$
p_{\left(N_{1}(d) \mid E_{1}(d)\right)}(n)=\frac{\left(\lambda l_{0} d\right)^{n} e^{-\lambda l_{0} d}}{n !\left(1-e^{-\lambda l_{0} d}\right)} .
$$

From the above,

$$
f_{B_{1} \mid E_{1}(d)}(x)= \begin{cases}\frac{\lambda l_{0} e^{-l_{0} \lambda x}}{1-e^{-\lambda l_{0} d}} & \text { if } x<d \\ 0 & \text { otherwise }\end{cases}
$$

As before, we obtain the marginal density of $B_{i}$ by taking the limit as $d \rightarrow \infty$.

$$
f_{B_{1}}(x)=\lambda l_{0} e^{-l_{0} \lambda x}
$$

Clearly, $B_{2}$ and $B_{3}$ will be identically distributed. Further, given that there are $N_{2}(d)$ sensors in $W_{2}(d)$, they will be independently and uniformly distributed in the region. This means that their distance from the origin, which is also their shortest distance to $L$, will have a density $\frac{2 x}{d^{2}}$ in $[0, d]$ and 0 outside. As in the case of $B_{1}$, we obtain $f_{B_{2}}(x)$ by first conditioning on $N_{2}(d), E_{2}(d)$ and evaluating the marginal. This turns out to be

$$
f_{B_{3}}(x)=f_{B_{2}}(x)=\lambda \pi x e^{-\frac{\lambda \pi x^{2}}{2}} .
$$

Recall that support $S u(L)$ is the maximum euclidean distance of the closest sensor from the path $L$. In the case of straight line paths, this will essentially be the distance of the closest sensor to the farthest end point of the straight line.

Let $E_{c}$ denote the event that the closest sensor is in $W_{1}$ and $E_{c}^{c}$ the complement event. The density of the support, $f_{S u(L)}(x)$, can therefore be obtained as

$$
f_{S u(L)}(x)=\operatorname{Pr}\left(E_{c}\right) f_{S u(L) \mid E_{c}}(x)+\operatorname{Pr}\left(E_{c}^{c}\right) f_{S u(L) \mid E_{c}^{c}}(x) .
$$

The event $E_{c}$ is the event that $B_{1}$ is less than $B_{2}$ and $B_{3}$. Therefore,

$$
\operatorname{Pr}\left(E_{c}\right)=\int_{x=0}^{\infty} f_{B_{1}}(x)\left(1-F_{B_{2}}(x)\right)\left(1-F_{B_{3}}(x)\right) d x .
$$

Also, given $E_{c}, S u(L)=\sqrt{B_{1}^{2}+\bar{T}^{2}}$. Here, $\bar{T}$ is the distance of the point $\bar{X}$, the projection of the closest sensor onto $\mathcal{L}$, to the farthest end point of $L$ (see Fig. 9). Since the sensors are deployed as a homogeneous Poisson process, $\bar{X}$ is uniformly distributed in $\left[0, l_{0}\right]$, which means $\bar{T}$ is uniformly distributed in $\left[\frac{l_{0}}{2}, l_{0}\right]$. Therefore,

$$
f_{S u(L) \mid E_{c}}(x)=\int_{l_{0} / 2}^{x} \frac{2 x f_{B_{1}}\left(\sqrt{x^{2}-t^{2}}\right)}{l_{0} \sqrt{x^{2}-t^{2}}} d t .
$$

In the case when the closest is in $W_{2}$ or $W_{3}$, the support can be written as

$$
S u(L)=\sqrt{\left(B_{2} \sin (\theta)\right)^{2}+\left(l_{0}+B_{2} \cos (\theta)\right)^{2}} .
$$

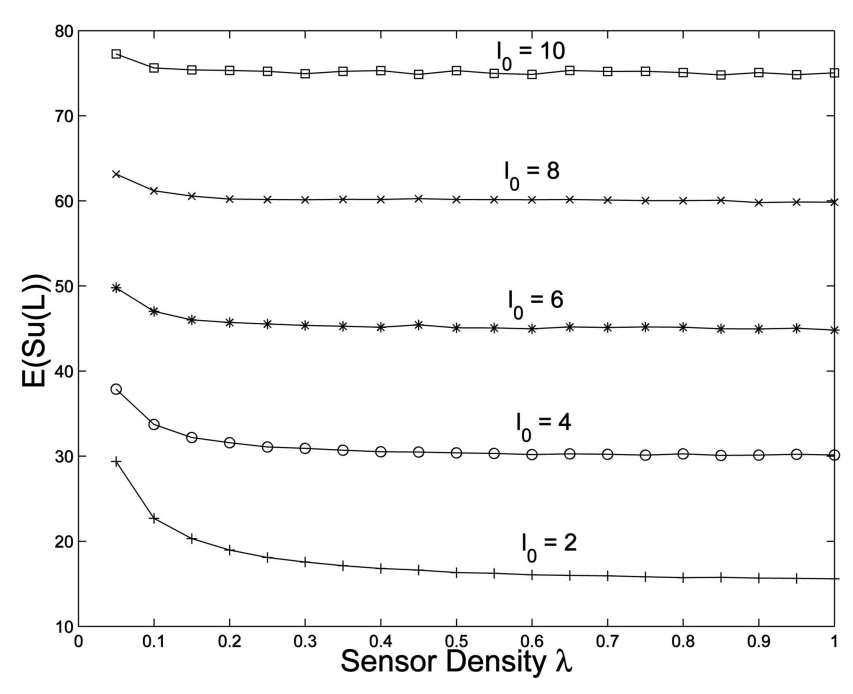

Fig. 15. Plot of $\mathrm{E}(S u(L))$ against $\lambda$ for different values of $l_{0}$.

Here, $\theta$ is the angle made by the line joining the closest sensor to the closest end of $L$. Since the sensor will be uniformly distributed in the semicircular regions, $\theta$ will be uniform in $[0, \pi]$.

We have not been able to obtain a closed form expression for either the distribution or the moments of the support. We therefore perform simulations to obtain numerical results. Fig. 15 plots $\mathrm{E}(S u(L))$ against $\lambda$ for different $l_{0}$. The variation across $\lambda$ decreases as $l_{0}$ increases. Thus, for large $l_{0}$, the support is dominated by $l_{0}$ and it does not appear to be a very useful measure of the "goodness" of deployment.

\section{Generalizations}

So far, we have analyzed the thresholded sensing of straight line paths. In this section, we extend these results to more general settings.

\subsection{Paths and Networks in Higher Dimensions}

Our first generalization is to extend the results for networks and paths of higher dimensions. As an example, consider a network deployed in three-dimensions that is used to track the movement of a fleet of airborne objects, say, a flock of birds, and also to track a specific object or bird. Since the "cross section" of the fleet or flock will be significantly higher than a single object, we may treat the latter as a point object and the former as a planar set. To track the fleet, the three-dimensional sensor network must sense a "two-dimensional path" while, in tracking the individual element, a one-dimensional path must be sensed.

Consider an $m$-dimensional straight line path $L$. When $m=1, L$ is a straight line and, when $m=2$, it is a rectangle. For a general $m, L$ will be an $m$-dimensional hypercuboid. Let $\left\{X_{i}, C_{i}\right\}$ form an $n$-dimensional Boolean process. Here, $\left\{X_{i}\right\}$ is an $n$-dimensional Poisson process of intensity $\lambda, C_{i}$ is a hypersphere of radius $R_{i}$ and $R_{i}$ are i.i.d. random variables with density $f_{R}(\cdot)$ with support in $[0,1]$. We are interested in the coverage/sensing properties of $L$ by 
$\left\{X_{i}, C_{i}\right\}$. Let $\mathcal{L}$ be the plane obtained by extending $L$ along the $m$ directions in which it is has a nonzero measure. As before, we project marked points, i.e., a point at $X_{i}$, onto $\mathcal{L}$ if the set $X_{i}+C_{i}$ intersects $\mathcal{L}$. This results in a point process $\left\{\bar{X}_{i}\right\}$. Let $\bar{C}_{i}$ be the intersection of $X_{i}+C_{i}$ and $\mathcal{L}$.

The following results can be obtained as above. See the Appendix for the details of the proof.

Lemma 4. The point process, $\left\{\bar{X}_{i}\right\}$, on $\mathcal{L}$ is a Poisson process of intensity $2 \beta_{n-m} \lambda$, where $\beta_{n-m}=\mathrm{E}\left(R_{i}^{n-m}\right)$.

Lemma 5. The set $\bar{C}_{i}$ is an $m$-dimensional hypersphere centered at $\bar{X}_{i}$ and has a radius $\bar{R}_{i}$, where the density of $R_{i}$ is

$$
f_{\bar{R}_{i}}(\bar{r})= \begin{cases}\frac{(n-m) \bar{r}}{\beta_{n-m}} \times \\ \int_{\bar{r}}^{1} f_{R}(r)\left(r^{2}-\bar{r}^{2}\right)^{\frac{n-m-2}{2}} d r & \text { if } 0 \leq \bar{r} \leq 1 \\ 0 & \text { otherwise. }\end{cases}
$$

Theorem 7. The projected point process $\left\{\bar{X}_{i}\right\}$ and the sets $\left\{\bar{C}_{i}\right\}$ constitute an $m$-dimensional Boolean process.

\subsection{Nonthresholded Sensing}

Recall that, in nonthresholded sensing, $\phi\left(X_{i}, x\right)$ is a continuous function that decreases as the distance of the point from the sensor increases. In general, $\phi\left(X_{i}, x\right)$ is taken to be $\frac{\eta}{d\left(X_{i}, x\right)^{\gamma}}$ and a point is considered sensed only if $\sum_{i} \frac{\eta}{d\left(X_{i}, x\right)^{\gamma}} \geq \theta$. Here, $d\left(X_{i}, x\right)$ is the euclidean distance between $X_{i}$ and $x$.

Recall that breach and support are not dependent on the sensing model and will remain the same. Further, it is shown in [4] that the region sensed by a network under the 1-thresholded model with the sensing radii is fixed at $\left(\frac{\eta}{\theta}\right)^{1 / \gamma}$ will be a subset of the region sensed by the network when the sensing is nonthresholded. Therefore, the exposure and the clump lengths for the nonthresholded model are lower bounded while the length to first sense is upper bounded by their values obtained for 1-thresholded sensing.

\subsection{Finite Operational Area}

Consider a square $A$ in which sensors are deployed in a Poisson manner, i.e., the number of sensors in $R \subset A$ is a Poisson random variable and is independent of the number of sensors in any nonoverlapping region. Let $P$ be a straight line path with the two end points on opposite edges of $A$. Since the sensors are distributed only inside $A$, the results we have derived above upper bound the sensing properties of $P$. Further, the results derived in the limit $\delta \rightarrow 0$ hold, as in that limit, even if sensors are deployed outside $A$, the length sensed by these sensors will be negligible.

\section{Discussion}

We have analyzed the properties of a two-dimensional Poisson sensor network in regard to its ability to track a path in the field. We obtain asymptotic and finite network statistics. We have shown that the fraction of a path that is 1-sensed is the same as the fraction of an area that is 1-sensed. It is not clear if this result carries over to $k>1$ and we leave that question open.

\section{APPENDIX A}

\section{Proof of Lemma 4}

Proof. Consider a differential element $d S$ on $\mathcal{L}$ of $m$-dimensional volume $d V$. The process $\left\{\bar{X}_{i}\right\}$ is a Poisson point process on $\mathcal{L}$ if the probability there is a projected point, i.e., $\bar{X}_{i}$ for some $i$ in $d S$ is $\bar{\lambda} d V$, and the arrivals also have the independent increments property.

There is a projected point in $d S$ if there is a corresponding $X$ in the $n$-dimensional differential element, $d P$, centered at $d S$ and of a 2 units length along the $n-m$ directions that are perpendicular to $\mathcal{L}$. The probability of this event is the product of the probability that there is a sensor in $d P$ (which is $\lambda d V+o(d V)$ ) and the probability that this sensor is marked. We calculate this next.

Given that a sensor (say, $s$ of sensing radius $R$ ) is in $d P$, it will be uniformly distributed there and the perpendicular distance of $s$ from $\mathcal{L}$, say, $Y$, will have a density

$$
f_{Y}(y)= \begin{cases}(n-m) y^{n-m-1} & 0 \leq y \leq 1 \\ 0 & \text { otherwise. }\end{cases}
$$

Therefore, the probability that $s$ is marked is

$$
\begin{aligned}
\operatorname{Pr}(s \text { is marked }) & =\int_{0}^{1} \operatorname{Pr}(Y \leq R) f_{R}(r) d r \\
& =\int_{0}^{1} r^{n-m} f_{R}(r) d r=\beta_{n-m} .
\end{aligned}
$$

The argument for independent increment property is identical to the argument in proof of Lemma 1.

\section{APPENDIX B \\ Proof of Lemma 5}

Proof. Consider a marked sensor $S$ at $X$ with a sensing radius $R$. Let $Y$ be its perpendicular distance from $\mathcal{L}$. Note that as the sensor is marked $R>Y$.

The region on $\mathcal{L}$ sensed by $S$ will be the hypersphere with radius $\bar{R}=\sqrt{R^{2}-Y^{2}}$. As before, by a transformation of random variables we have the distribution function, $F_{\bar{R}}(\cdot)$, can be written as

$$
F_{\bar{R}}(\bar{r})=\int_{r, y: \sqrt{r^{2}-y^{2}}=\bar{r}} f_{(R, Y \mid R \geq Y)}(r, y) d r d y .
$$

The probability that a sensor, which is within a unit distance from $\mathcal{L}$, is marked is $\beta_{n-m}$ (proof of Lemma 4 ). Further, $Y$ is independent of $R$ and its is density is given by (9). Therefore,

$$
f_{(R, Y \mid R>Y)}(r, y)=\frac{(n-m) f_{R}(r) y^{n-m-1}}{\beta_{m-n}} .
$$

Substituting for $f_{(R, Y \mid R>Y)}(r, y)$ in (10) and differentiating with respect to $\bar{r}$, we get

$$
f_{\bar{R}_{i}}(\bar{r})= \begin{cases}\frac{(n-m) \bar{r}}{\beta_{n-m}} \times & \\ \int_{\bar{r}}^{1} f_{R}(r)\left(r^{2}-\bar{r}^{2}\right)^{\frac{n-m-2}{2}} d r & \text { if } 0 \leq \bar{r} \leq 1 \\ 0 & \text { otherwise }\end{cases}
$$




\section{REFERENCES}

[1] S. Megerian, F. Koushanfar, M. Potkonjak, and M.B. Sivastava, "Worst-Case and Best-Case Coverage in Sensor Networks," IEEE Trans. Mobile Computing, vol. 4, no. 1, pp. 84-92, Jan./Feb. 2005.

[2] J. Aslam et al., "Tracking a Moving Object with a Binary Sensor Network," Proc. First Int'l Conf. Embedded Networked Sensor Systems, 2003.

[3] R.R. Brooks, P. Ramanathan, and A.M. Sayeed, "Distributed Target Classification and Tracking in Sensor Networks," Proc. IEEE, 2003.

[4] B. Liu and D. Towsley, "A Study on the Coverage of Large Scale Networks," Proc. ACM MobiHoc, 2004

[5] H. Zhang and J. Hou, "On Deriving the Upper Bound of $\alpha$-Lifetime for Large Sensor Networks," Proc. ACM MobiHoc, 2004.

[6] S. Kumar, T.H. Lai, and J. Balogh, "On $k$-Coverage in a Mostly Sleeping Sensor Network," Proc. 10th Ann. Int'l Conf. Mobile Computing and Networking, 2004.

[7] P.J. Wan and C.W. Yi, "Coverage by Randomly Deployed Wireless Sensor Networks," Proc. Fourth IEEE Int'l Symp. Network Computing and Applications, 2005.

[8] P. Hall, Introduction to the Theory of Coverage Process. John Wiley and Sons, 1988.

[9] S. Megerian et al., "Exposure in Wireless Sensor Networks: Theory and Practical Solutions," J. Wireless Networks, vol. 8, 2002

[10] S. Athreya, R. Roy, and A. Sarkar, "On the Coverage of Space by Random Sets," Advances in Applied Probability, vol. 36, pp. 1-18, 2004.

[11] X.Y. Li, P.J. Wan, and O. Frieder, "Coverage in Wireless Ad-Hoc Sensor Networks," IEEE Trans. Computers, vol. 52, no. 6, pp. 753763, June 2003.

[12] Q. Huang, "Solving an Open Sensor Exposure Problem Using Variational Calculus," www.cse.seas.wustl.edu/techreportfiles/ getreport.asp?237, 2003.

[13] T. Clouqueur, V. Phipatanasuphorn, P. Ramanathan, and K Saluja, "Sensor Deployment Strategy for Source Tracking," Proc. First ACM Int'l Workshop Wireless Sensor Networks and Applications, 2002.

[14] X. Wang et al., "Integrated Coverage and Connectivity Configuration in Wireless Sensor Networks," Proc. First ACM Conf. Embedded Networked Sensor Systems, 2003.

[15] H. Zhang and J. Hou, "Maintaining Sensing Coverage and Connectivity in Larger Sensor Networks," Ad Hoc and Sensor Wireless Networks: An Int'l J., vol. 1, no. 1, pp. 89-123, 2005.
S. Sundhar Ram received the BTech and MTech degrees, both in 2006, from the Indian Institute of Technology, Bombay, India, in electrical engineering. $\mathrm{He}$ is currently pursuing the $\mathrm{PhD}$ degree in electrical engineering at the University of Illinois Urbana-Champaign. $\mathrm{He}$ is a student member of the IEEE.

D. Manjunath received the BE degree from Mysore University, the MS degree from the Indian Institute of Technology, Madras, and the PhD degree from Rensselaer Polytechnic Institute, Troy, New York, in 1986, 1989, and 1993, respectively. He has worked in the Corporate R \& D Center of General Electric in Scehenectady, New York (1990), in the Computer and Information Sciences Department at the University of Delaware (1992-1993), and in the Computer Science Department at the University of Toronto (1993-1984). He was on the electrical engineering faculty of the Indian Institute of Technology, Kanpur, during 1994-1998. $\mathrm{He}$ has been with the electrical engineering Department of IIT-Bombay since July 1998, where is now an associate professor. His research interests are in the areas of communication networks, performance analysis of systems, queuing systems, and multimedia communications. His recent research has been in the areas of network traffic and performance measurement, analysis of random wireless data and sensor networks, network pricing, and queue control. He is the coauthor of the textbook Communication Networking: An Analytical Approach (Morgan-Kaufman, May 2004). He is a member of the IEEE.

Srikanth K. lyer received the MSc (5yr Integrated) degree from the Indian Institute of Technology (IIT) Kanpur and the MS and PhD degrees from the University of California at Santa Barbara in 1990, 1991, and 1994, respectively. He did his postdoctoral work at the Technion, Israel, and the University of North Carolina, Chapel Hill, from 1994-1996. He was on the faculty of the Department of Mathematics at the Indian Institute of Technology Kanpur from 1996-2004. He has been with the Department of Mathematics at the Indian Institute of Science, Bangalore, since December 2004, where he is now an associate professor. His reseach interests are branching-diffusing particle systems, random geometric graphs, coverage and tracking problems, correlated queues, and mathematical finance.

D. Yogeshwaran received the BSc (mathematics) degree from the Sri Sathya Sai Institute of Higher Learning in 2003 and the MS (mathematics) degree from the Indian Institute of Science in 2006. He has worked on random geometric graphs and coverage processes with applications to wireless sensor networks for his master's thesis. He was also a summer intern at ENS DI TREC, Paris (an INRIA research group on network theory and communications). His research interests are applied probability and stochastic processes. 